\title{
LA CALIDAD DE LA EDUCACIÓN SUPERIOR Y SU ACREDITACIÓN: LA EXPERIENCIA CENTROAMERICANA
}

\author{
CARLos TÜNNERMANn Bernheim*
}

\begin{abstract}
* Ha sido Rector de la Universidad Nacional Autónoma de Nicaragua por tres períodos (1964-1974); Ministro de Educación (1979-1984). Miembro del Consejo Ejecutivo de la UNESCO (1990-1994), Consejero Especial del Director General de la UNESCO; miembro del Consejo de Administración de la Universidad de las Naciones Unidas (Tokyo, Japón); de la Junta Directiva de la Asociación Internacional de Universidades y Presidente en dos ocasiones de la Unión de Universidades de América Latina (UDUAL). Es autor de numerosos libros sobre educación superior. ctunnermann@yahoo.com.
\end{abstract}

Resumen: Este capítulo presenta un estudio comparado de los procesos de acreditación en América Central $\mathrm{y}$ de las instituciones que desarrollan esta tarea.

Palabras claves: Educación Superior. Acreditación de la Educación Superior. Evaluación. Sistemas nacionales de acreditación. Sistema centroamericano de acreditación.

Resumo: Este capítulo apresenta um estudo comparado dos processos de acreditação na América Central e das IES que desenvolvem essa tarefa.

Palavras-chave: Educação Superior. Acreditação da Educação superior. Avaliação. Sistemas nacionais de acreditação. Sistema centroamericano de acreditação.

Abstract: This chapter presents a comparative study of the accreditation processes in Central America and of the institutions that are responsible for developing them.

Keywords: Higher education. Higher education accreditation. Evaluation. National accreditation systems. Central American accreditation system.

\section{La acreditación. su importancia en el actual contexto internacional.}

En el contexto de la sociedad contemporánea, las instituciones de educación superior son parte del mundo académico global. Hoy en día, ninguna universidad puede sustraerse de las relaciones internacionales y los intercambios académicos con el extranjero. Sin embargo, cuando nos referimos a la internacionalización de la educación superior estamos hablando de algo que va más allá del fomento de las relaciones internacionales o de la simple promoción de la cooperación internacional. En realidad, nos estamos refiriendo a una nueva función de la universidad contemporánea, que viene a agregarse a sus funciones clásicas y que consiste fundamentalmente en subrayar la dimensión internacional de su quehacer. Como señalan algunos autores, la internacionalización de la educación superior es la respuesta construida por los universitarios frente al fenómeno de la globalización y de la naturaleza sin fronteras del conocimiento 
contemporáneo. Las universidades, en sus orígenes, nacieron como instituciones inter-naciones, de manera que la internacionalización se aviene con su naturaleza primigenia.

De esta manera, existe hoy en día una dimensión internacional en la educación superior. Esta dimensión es la que hace indispensable la existencia de sistemas de acreditación, a fin de establecer los mecanismos adecuados para el aseguramiento de la calidad no solo de los ofrecimientos de educación superior a nivel nacional, sino también de aquellos que desbordan las fronteras nacionales.

No cabe confundir internacionalización de la educación superior con transnacionalización del sector educativo de tercer nivel, que conlleva su transformación en un servicio sujeto a las reglas del mercado, con predominio de los intereses de las empresas educativas transnacionales. Mientras en la internacionalización se propugna, siguiendo los lineamientos de la Declaración Mundial sobre la Educación Superior, por una cooperación internacional solidaria con énfasis en la cooperación horizontal, basada en el diálogo intercultural y respetuosa de la idiosincracia e identidad de los países participantes, así como el diseño de redes interuniversitarias y de espacios académicos ampliados, en la transnacionalización se trata de facilitar el establecimiento en nuestros países de filiales de Universidades extranjeras, de una cooperación dominada aun por criterios asistenciales, así como la venta de franquicias académicas, la creación de universidades corporativas, auspiciadas por las grandes empresas transnacionales, los programas multimedios y las universidades virtuales, controladas por universidades y empresas de los países más desarrollados. Este nuevo panorama que comienza a configurarse en nuestros países, ha hecho surgir voces de alerta por el peligro que representan para nuestra soberanía e identidad y refuerzan la necesidad de contar con sistemas nacionales de acreditación.

En la internacionalización los instrumentos para su promoción suelen ser las redes académicas, los hermanamientos solidarios entre universidades, la cooperación horizontal, la creación de espacios académicos ampliados, etc.

El concepto clave para resguardar, en un mundo globalizado y de mercados abiertos y competitivos, la autonomía, la libertad de cátedras y los principios esenciales que caracterizan el quehacer universitario, tal como hasta ahora lo hemos conocido, es el criterio proclamado por la aludida Declaración Mundial sobre la Educación Superior, que nítidamente define la educación superior como un bien público y el conocimiento generado en ella como un bien social al servicio de la humanidad. 
En recientes reuniones de académicos latinoamericanos, se ha advertido el peligro que representa la pretensión de aplicar a la educación superior las normas del mercado, que al asumirla como un simple bien transable la reducen al nivel de mercancía. El riesgo mayor proviene, en estos momentos, de la resolución adoptada en el año 2002 por la Organización Mundial de Comercio (OMC), de incluir la educación superior como un servicio comercial regulado en el marco del Acuerdo General de Comercio de Servicios (GATS, por su sigla en inglés), decisión que han impugnado, entre otros organismos, la Asociación Internacional de Universidades (AIU), la Unión de Universidades de América Latina (UDUAL), la Asociación de Universidades y Colegios de Canadá, la Asociación de Universidades Europeas, el American Council on Education, la Asociación de Universidades del Grupo de Montevideo, el Consejo Superior Universitario Centroamericano (CSUCA) y las Cumbres Iberoamericanas de Rectores de Universidades Públicas.

Tampoco es aceptable la pretensión de declarar a la educación superior como "un bien público global", pues el calificativo de global se presta a que no esté sujeta, en cada Estado, a las normas y regulaciones que soberanamente establezca cada país, y es una manera sutil de empujar a la educación superior a la órbita de la OMC, sacándola de la jurisdicción de los estados nacionales, y por lo mismo, renunciando estos a toda regulación o normativa que impida su libre circulación y comercio.

No se trata de rechazar por rechazar los programas de proveedores extranjeros, desde luego que hay campos especializados en que pueden contribuir a completar las ofertas académicas nacionales, pero nada ganan nuestros países con complementar esas ofertas con programas mediocres, de pésima calidad. Tampoco nos conviene la competencia asimétrica y, mucho menos, la desleal.

En este sentido, es urgente incorporar en nuestros Sistemas Nacionales y Regionales de Acreditación, las disposiciones pertinentes que extiendan la competencia de nuestros sistemas a la acreditación de los programas de los proveedores extranjeros que se ofrezcan en nuestros países, ya sean estos presenciales, a distancia o virtuales. El establecimiento de estándares para evaluar esos ofrecimientos es de gran importancia y necesidad. Por lo general, nuestros sistemas de acreditación no contemplan estos ofrecimientos transfronterizos. También tenemos que estar prevenidos sobre el hecho de que no solo nos venden cursos virtuales enlatados y otros insumos educativos de baja calidad, sino que también estén apareciendo agencias acreditadoras extranjeras que prácticamente venden servicios de acreditación, especialmente a proveedores extranjeros. 
Nuestros sistemas de acreditación no siempre incluyen estándares e indicadores para evaluar y acreditar, los programas de educación a distancia ni siquiera para los que se imparten a nivel nacional $\mathrm{y}$, menos aún, para los programas virtuales. Pero el vacío mayor es en relación a los ofrecimientos hechos por proveedores externos.Del debate se desprende que las respuestas a los riesgos que significa el GATS para la Educación Superior, corresponden principalmente al ámbito político: la necesidad que los Estados tienen de crear Sistemas Nacionales de Acreditación sólidos, transparentes, que gocen de gran credibilidad y que incorporen normas para la acreditación de programas ofrecidos por proveedores extranjeros, es decir todas la modalidades llamadas "Crossborders" o transfronterizos. Estas normas deberán hacerse extensivas a la autorización para el funcionamiento de agencias de acreditación extranjeras. Se pregunta Jane Knight: “¿Tienen los países la capacidad y voluntad política de establecer y supervisar sistemas de aseguramiento de la calidad de los programas de educación superior, tanto para la exportación como la importación?". Para dar una respuesta positiva a esta interrogante, los Estados deben incorporar en sus legislaciones normas relacionadas con la acreditación de programas a distancia, "on-line", ofrecimientos transfronterizos de educación superior, de cualquier tipo, y de las agencias extranjeras que pretendan llevar a cabo acreditaciones en sus territorios.

Convendría, además, promover la existencia de organismos subregionales oficialmente autorizados para acreditar agencias de acreditación, lo cual permitiría una mayor movilidad de estudiantes, profesionales y profesores entre las subregiones. Una contribución valiosa en este sentido sería aportar criterios para la definición de estándares subregionales que, eventualmente lleven a la definición de estándares y principios de buenas prácticas, a nivel regional.

\section{La experiencia centroamericana. Procesos de autoevaluación institucional y sistemas nacionales y regionales de acreditación.}

\subsection{Antecedentes.}

En Centroamérica, el Consejo Superior Universitario Centroamericano (CSUCA) ha sido el organismo pionero no solo de la integración regional de la educación superior sino también de los procesos de aseguramiento de la

1 Jane Knight: "El comercio de la educación superior. Nuevas oportunidades y riesgos potenciales" en revista Foreign Affairs en español, Vol. 5 Núm. 2, 2005, p. 34 y sigts. 
calidad académica. El Primer "Plan para la integración regional de la educación superior centroamericana", aprobado por las universidades miembros del CSUCA en 1962, contiene algunos acuerdos encaminados a garantizar la calidad académica, como son el referente a los requisitos mínimos para el establecimiento de cursos de postgrado y el acuerdo que estableció los requisitos que los programas de pregrado y postgrado debían cumplir para obtener su reconocimiento como programas al servicio de toda la región, es decir, la regionalidad.

Mencionamos estos antecedentes para que se vea que, desde hace más de cuatro décadas, el tema de la calidad ha estado presente en la agenda de la educación superior centroamericana, lo cual ha conducido a la creación, en 1962, del Sistema de Carreras y Postgrados Regionales (SICAR) y, a partir de 1998, del Sistema Centroamericano de Evaluación y Acreditación de la Educación Superior (SICEVAES).

\subsection{El sistema centroamericano de evaluación y acreditación de la educación superior (SICEVAES).}

E1 SICEVAES fue creado por el CSUCA en 1998 y es el resultado de un largo proceso de concertación encaminado a lograr la homologación de los diplomas y títulos profesionales en Centroamérica, a fin de promover la movilidad de los graduados universitarios en la región. En el proceso se llegó a la conclusión que el camino más expedito para lograr esa homologación y movilidad académica era a través del establecimiento de sistemas de evaluación y acreditación.

En el IV Congreso Universitario Centroamericano (1995) se aprobó el Segundo Programa Regional de Integración de la Educación Superior Centroamericana (PIRESC II) que incluye, como área prioritaria de trabajo del CSUCA la Evaluación y Acreditación Universitaria. Se contempla también, como parte del Plan, la creación de un Sistema Centroamericano de Evaluación y Acreditación Universitaria.

Ese mismo año, el CSUCA elaboró una primera propuesta acerca de la posibilidad de crear en Centroamérica un sistema de acreditación de alcances regionales. El proyecto fue finalmente aprobado por el CSUCA en su LVI Reunión, celebrada en Panamá en septiembre de 1998, con el nombre de Sistema Centroamericano de Evaluación y Acreditación de la Educación Superior (SICEVAES).

El CSUCA señaló al SICEVAES, entre otros, los objetivos siguientes:

" - Fomentar en las universidades centroamericanas una cultura de calidad orientada al mejoramiento de las carreras. 
- Lograr consenso entre las universidades centroamericanas sobre los referentes para evaluar la calidad de carreras de educación superior.

- Promover procesos de autoevaluación de carreras con fines de mejoramiento y acreditación.

- Realizar procesos de evaluación externa por pares académicos con fines de mejoramiento de las carreras.

- Promover la formulación de planes de mejoramiento para superar los problemas, debilidades y carencias identificadas en los procesos de autoevaluación y evaluación externa de carreras".

En su primera etapa el SICEVAES promovió la evaluación de carreras con fines de mejoramiento de la calidad. Posteriormente el Consejo Superior Universitario Centroamericano, con el propósito de cumplir con los objetivos definidos en la creación del sistema, tomó el acuerdo de establecer las condiciones para el desarrollo de procesos de autoevaluación que además de responder al propósito interno del mejoramiento atendieran al propósito externo de la acreditación.

El SICEVAES fue objeto, en el año 2005, de una cuidadosa metaevaluación, que comprobó el éxito del programa y el logro de sus principales objetivos. La existencia del SICEVAES ha permitido que aproximadamente un centenar de programas ofrecidos por universidades estatales miembros del CSUCA hayan completado, o estén a punto de completar, procesos de autoevaluación, de los cuales 31 han recibido las visitas de pares externos. Además, y siempre a través del SICEVAES, se llevaron a cabo dos procesos de autoevaluación institucional en Panamá.

\subsection{El sistema de carreras y postgrados regionales centroamericanos (SICAR).}

El SICAR tiene una larga historia, que arranca del "Primer Plan para la integración regional de la educación superior centroamericana”, aprobado por el CSUCA en 1962. Ese mismo año se aprobaron las primeras carreras regionales, conforme las normas y requisitos para establecer este tipo de carreras aprobadas el año anterior.

En la LVI Sesión Ordinaria (septiembre de 1998), el CSUCA aprobó los cinco documentos que redefinen, reestructuran y reorientan al SICAR:

1. Convenio para garantizar el funcionamiento del Sistema de Carreras y Posgrados Regionales Centroamericanos (SICAR). 
2. Reglamento del Sistema de Carreras y Programas Regionales Centroamericanos (SICAR).

3. Conceptos Básicos y Normas Académicas de los Programas Regionales Centroamericanos.

4. Normas y Procedimientos para acreditar la categoría regional centroamericana de carreras, programas y cursos especiales de posgrado.

5. Programa centroamericano de intercambio académico.

En su conjunto estos documentos marcan un hito en el desarrollo histórico y brindan una nueva perspectiva para el desarrollo del Sistema de Carreras y Posgrados Regionales del CSUCA. Hoy día el SICAR es un sistema establecido $\mathrm{y}$ en pleno funcionamiento.

Del 29 de septiembre al $1^{\circ}$ de octubre de 2005, el CSUCA y el CCA auspiciaron un Seminario-Taller sobre Acreditación de Calidad de Programas de Postgrado, en la ciudad de Guatemala. Este Seminario tenía, entre sus objetivos, "promover la creación de la Comisión Centroamericana de Acreditación de Programas de Postgrado, con la participación de distintos sectores e instituciones legítimamente interesada en la calidad y pertinencia de la educación superior de postgrado de Centroamérica".

Se conoció en este taller una propuesta para crear la Comisión Centroamericana de Acreditación de Programas de Postgrados que tendría a su cargo "dar fe pública internacional de la calidad de los programas de postgrado de Centroamérica, con autonomía, transparencia, ética y participación internacional del más alto nivel para el mejoramiento continuo y la integración regional". Como resultado del taller, quedó constituida una Comisión Centroamericana de Acreditación de Programas de Postgrado que siguió trabajando sobre la propuesta presentada por el CSUCA.

Finalmente, en el mes de agosto de 2006, se firmó en Tegucigalpa, Honduras el Convenio Constitutivo de la "Agencia Centroamericana de Acreditación de Programas de Postgrado” y quedaron aprobados sus Estatutos.

Establecer en Centroamérica un mecanismo transparente y confiable de acreditación de los postgrados era una necesidad, ya que es en este nivel donde más se hacen sentir los fenómenos contemporáneos de la internacionalización de la educación superior y la globalización del conocimiento. La oferta de postgrados en nuestra región no solo proviene de nuestras propias universidades, públicas y privadas, sino también de una multitud de oferentes y proveedores de otros países, a través de distintas modalidades, presenciales, a distancia y virtual. La ACAP tendrá a su cargo la acreditación de todos los ofrecimientos de educación superior que se realicen en Centroamérica, al nivel de postgrado. 


\subsection{Los sistemas nacionales de acreditación de la educacion superior en Centroamérica. Situación actual por países.}

\subsubsection{Costa Rica:}

En este país han sido las propias universidades, asociadas en el Consejo Nacional de Rectores (CONARE) las que en virtud de un Convenio crearon el Sistema Nacional de Acreditación de la Educación Superior (SINAES), en pleno ejercicio de su autonomía. En efecto, el CONARE, en su sesión celebrada el 23 de marzo de 1993 (Sesión N07-93) aprobó el Convenio para la creación del SINAES, que no comenzó a funcionar sino hasta en 1998, cuando el Convenio Constitutivo fue ratificado por las cuatro universidades públicas y cuatro privadas (las de mayor prestigio del país). En virtud de la Ley 8256 del 17 de mayo de 2002, la Asamblea Nacional de Costa Rica reconoció al SINAES como el ente nacional oficial de acreditación. Los tres objetivos fundamentales del SINAES son: (a) "Coadyuvar al logro de los principios de excelencia establecidos en la legislación nacional y al esfuerzo que realizan las instituciones universitarias por mejorar la calidad de los programas y carreras que ofrecen; (b) mostrar la conveniencia que tiene para las instituciones universitarias someterse a un proceso de acreditación y propiciar la confianza de la sociedad costarricense en los programas y carreras acreditados, orientándola también con respecto a la calidad de las diferentes opciones de educación superior; (c) certificar el nivel de calidad de las carreras y de los programas sometidos a acreditación, garantizando la eficiencia, calidad de criterios y estándares aplicados en ese proceso".

El SINAES está dirigido por un Consejo integrado por ocho miembros: Cuatro son designados por las universidades estatales y los otros cuatro por las universidades privadas. Una vez nombrados, por consenso entre las universidades que representan, los miembros del Consejo gozan de plena independencia de criterio en el ejercicio de su función. Duran en sus cargos cuatro años, pudiendo ser reelectos por períodos iguales y sucesivos en forma indefinida. El Consejo tiene, entre otras funciones, las siguientes: (a) atender y acreditar programas y carreras, respetando la individualidad, autonomía y fines de la institución universitaria solicitante; (b) elaborar, aprobar, actualizar y vigilar el estricto cumplimiento de los procedimientos, estándares y criterios de evaluación aplicables a la acreditación, la autorregulación y la autoevaluación; (c) realizar, por lo menos cada cinco años, un 
proceso de autoevaluación de criterios, procedimientos y estándares empleados en los trámites de acreditación, con la supervisión de un organismo externo especializado en la materia; (d) mantener informadas a las instituciones universitarias y a la comunidad nacional acerca de los procedimientos y criterios que se emplean en los trámites de acreditación y cualquier otro aspecto de interés general; y (e) informar al Consejo Nacional de Rectores el resultado de los estudios de acreditación realizados a las universidades estatales y al Consejo Nacional de Enseñanza Superior Universitaria Privada.

En relación con el SINAES conviene destacar lo siguiente:

- El SINAES acredita la calidad de los programas y carreras de las instituciones de Educación Superior, tanto públicas como privadas. El SINAES, no acredita instituciones ni unidades académicas como facultades, escuelas o departamentos.

- Se financia con aportes de las Universidades públicas y privadas, donaciones e ingresos propios. La Acreditación es un proceso voluntario.

- El propósito de la acreditación es identificar carreras y programas que cumplan con los requisitos de calidad establecidos por el SINAES, y con ello mejorar la calidad de los programas y carreras, y garantizar públicamente la calidad de estos.

- ¿Cuáles son los componentes que se evalúan? Personal académico, currículum, estudiantes, infraestructura y equipamiento, administración, el impacto y pertinencia de la carrera.

- Estándares: (i). Los profesores deben tener un nivel académico igual o superior al nivel en que imparten lecciones; (ii) Un 50\% debe tener un mínimo de tres años de experiencia docente universitaria; (iii) Un $50 \%$ debe tener un mínimo de tres años de experiencia profesional; (iv) Bajo ninguna circunstancia, la carrera o programa podrá depender de una sola persona.

- La evaluación se basa en la información detallada que proporciona la institución y en el juicio colectivo de los pares. Pero, la acreditación como tal es una decisión del Consejo del SINAES y estará fundamentada en criterios cuantitativos y cualitativos.

- La acreditación tiene un período de vigencia de 4 años. Puede, en casos especiales otorgarse por períodos menores o condicionada al 
cumplimiento de requerimientos específicos. La Acreditación se hace pública. El SINAES publica una lista de carreras acreditadas.

- El SINAES hace dos convocatorias por año: una en mayo y la segunda en octubre.

\subsubsection{El Salvador:}

La Ley de Educación Superior de El Salvador (1995) creó el Sistema de Supervisión y Mejoramiento de la Calidad Académica de la Educación Superior. Este Sistema comprende el proceso de certificación (anual), el de evaluación (bianual) y el de acreditación. Por decreto presidencial, dictado a través del Ministerio de Educación el 10 de marzo del 2000, se reglamentó la integración y funciones de la Comisión de Acreditación de la Calidad Académica de las Instituciones de Educación Superior. La Comisión está integrada por siete miembros y goza de autonomía de acción en su cometido. El subsistema de acreditación es un componente del Sistema de Supervisión y Mejoramiento de la Calidad Académica de la Educación Superior. Los miembros de la Comisión son nombrados de mutuo acuerdo entre el Ministerio de Educación y el Consejo de Educación Superior, que preside el Ministro de Educación y al cual pertenecen la Universidad de El Salvador y cuatro universidades privadas del país. Tienen derecho a solicitar acreditación todas las instituciones de educación superior legalmente establecidas. Las instituciones acreditadas tendrán acceso a programas de asistencia e incentivos fiscales. El Decreto establece dos categorías dentro del proceso de acreditación: las instituciones acreditadas, que serán aquellas que satisfagan todos los criterios de evaluación de la calidad establecidos por la Comisión, y las instituciones candidatas, que son las que aún no llenan todos los requisitos pero que tienen una alta probabilidad de cumplidos en un plazo determinado, que no puede ser mayor de dos años.

En relación con la Comisión de Acreditación de la Calidad Académica conviene destacar lo siguiente:

- La Comisión de Acreditación de la Calidad Académica se financia con recursos que le asigna el Ministerio de Educación, consignados en el presupuesto de la Dirección Nacional de Educación Superior del Ministerio. La Comisión se reúne ordinariamente una vez cada seis meses. Las sesiones duran un máximo de 3 días. 
- La solicitud de acreditación debe ir acompañada con tres ejemplares del autoestudio de las dos últimas evaluaciones obligatorias, así como de las correspondientes Resoluciones del Ministerio sobre esos informes. La Comisión pide, al mismo tiempo, a la Dirección Nacional de Educación Superior del Ministerio, un informe pormenorizado del expediente de la institución solicitante, que se agrega a la documentación.

- El expediente de la solicitud pasa a estudio de un miembro de la Comisión, designado por el Presidente en consulta con todos los miembros. El lector del expediente debe presentar un dictamen sobre el mismo en la sesión correspondiente, actuando como relator del expediente. La Comisión puede solicitar la presencia del representante legal de la Universidad y del Presidente de la Comisión de pares externos, a quienes escuchará separadamente. También la del Director Nacional de Educación Superior.

- La Resolución, debidamente fundamentada, no admite recurso alguno. Si la Resolución es positiva, el Ministerio de Educación emite el Acuerdo Ejecutivo de Acreditación.

- La Acreditación tiene validez por 5 años.

\subsubsection{Guatemala:}

"En Guatemala, la Universidad de San Carlos USAC, única universidad pública del país, goza de plena autonomía y se regula a si misma en el marco de lo establecido para ello en la Constitución de la República, su Ley Orgánica y Estatutos. Para la regulación de la educación superior privada existe el Consejo de Educación Privada Superior CEPS, que es la instancia legal que autoriza la creación de universidades privadas y supervisa el funcionamiento de las mismas para asegurar su calidad académica. El CEPS está constituido por 5 miembros titulares y cinco suplentes de los cuales el $40 \%$ son nombrados por el Consejo Superior Universitario de la Universidad de San Carlos de Guatemala, el otro 40\% por los Rectores del conjunto de las universidades privadas del país y el otro $20 \%$ por los Presidentes de los Colegios Profesionales del país. En Guatemala puede decirse que de hecho existen dos subsistemas de educación superior, el estatal (la USAC que se regula a si misma) y el privado (en cuya regulación también participa la USAC). El Ministerio de Educación no tiene ninguna participación en el funcionamiento de la 
educación superior del país. La ley otorga a las universidades privadas establecidas en Guatemala gran autonomía y libertad académica y a la vez delega en el CEPS la responsabilidad de velar porque se mantenga el nivel académico en las universidades privadas del país sin menoscabo de su independencia. La supervisión, que por definición le corresponde, solo se hace en caso de denuncias. Esto debido a la limitada infraestructura institucional de este organismo. El CEPS, para el mejor cumplimiento de su mandato ha decidido impulsar la creación de un Sistema Nacional de Acreditación de la Educación Privada Superior (SINADEPS), proyecto que se ha venido gestando en los últimos dos años. Por su parte los órganos directivos de la Universidad de San Carlos de Guatemala han venido impulsando entre sus unidades académicas los procesos de autoevaluación y evaluación externa para el mejoramiento de la calidad de carreras específicas, en el marco del Sistema Centroamericano de Evaluación y Acreditación de la Educación Superior SICEVAES, que desarrolla el CSUCA"².

\subsubsection{Honduras:}

“En Honduras, la Universidad Nacional Autónoma de Honduras UNAH goza de plena autonomía y se regula a si misma en el marco de lo establecido en la Constitución y en su Ley Orgánica. Asimismo, el Artículo 160 de la Constitución de la República de Honduras le otorga a la Universidad Nacional Autónoma, como organismo autónomo y descentralizado del Estado, la responsabilidad de autorizar, organizar, dirigir y desarrollar la educación superior y profesional del país. Este precepto constitucional es desarrollado por la Ley de Educación Superior de 1989 y su Reglamento. De acuerdo a esta Ley, la Educación Superior de Honduras es dirigida por el Consejo de Educación Superior, el cual está constituido por 14 miembros, 8 de los cuales son nombrados por la Universidad Nacional Autónoma de Honduras. Este Consejo es presidido por el Rector de la Universidad Nacional Autónoma y tiene un órgano ejecutivo, la Dirección de Educación Superior, el cual es nombrado por el claustro pleno de la Universidad Nacional Autónoma de Honduras. En este caso teóricamente existe un solo sistema Nacional de Educación Superior regulado por la Ley de Educación Superior y dirigido por un órgano nacional único (el Consejo de Educación Superior), no obstante en la práctica se afirma que

2 Francisco Alarcón Alba y Julio Guillermo Luna: Antecedentes, Situación Actual y perspectivas de la evaluación y acreditación de la educación superior en Centroamérica, Secretaría Permanente del CSUCA, Guatemala, mayo de 2003 / (fotocopiado). 
existen dos subsistemas, la Universidad Nacional Autónoma de Honduras por un lado y el resto de instituciones (públicas y privadas) que existen en Honduras por el otro. En cualquier caso, en Honduras la Secretaría de Educación (Ministerio) no tiene ninguna participación en la regulación de la educación superior del país. La Universidad Nacional Autónoma de Honduras, por medio del Consejo de Educación Superior como órgano de dirección y decisión del sistema, emitió NORMAS ACADEMICAS DE LA EDUCACION SUPERIOR con el fin de regular el desarrollo de la educación superior de Honduras. Estas normas establecen un marco filosófico, conceptual y metodológico general y definen los estándares generales que las instituciones de educación superior de Honduras han de cumplir. No obstante, hasta hace muy recientemente en Honduras no había existido iniciativa conocida para el establecimiento de un sistema nacional de acreditación de la calidad de la educación superior, ni políticas nacionales para el impulso de la acreditación. No obstante ahora que conjuntamente se ha definido que el Consejo Centroamericano de Acreditación CCA no realizará directamente acreditación de programas e instituciones de educación superior, sino que funcionará como un organismo acreditador de segundo nivel, es decir acreditador de acreditadores, los rectores y dirigentes de colegios profesionales de Honduras se están planteando la necesidad de, promover la creación de un sistema nacional hondureño de acreditación que sea acreditado por el CCA"3.

El CCA ha brindado asesoría técnica a la Comisión creada por el Consejo de Educación Superior para elaborar un Marco de referencia y los principios básicos para crear un Sistema Nacional de Acreditación en Honduras.

\subsubsection{Panamá:}

“En Panamá, al igual que en los demás países de la región, las universidades estatales gozan de gran autonomía por lo que tienen la plena potestad de regularse a si mismas, en el marco de lo que al respecto establece la Constitución Política y sus Leyes constitutivas respectivas. Las universidades estatales no tienen una instancia de coordinación y autorregulación conjunta como ocurre en Costa Rica sino que se regulan a si mismas de manera independiente. Por otra parte, la autorización de la creación de universidades privadas en Panamá se realiza mediante Decretos Ejecutivos como un acto conjunto del Ministerio de Educación y la

3 Francisco Alarcón, etc: Ibídem. 
Presidencia de la República. No obstante el artículo 95 de la Constitución Política de Panamá establece que “...la Universidad Oficial del Estado fiscalizará a las universidades particulares aprobadas oficialmente para garantizar los títulos que se expiden y revalidará los de universidades extranjeras en los casos que la Ley establezca." El papel fiscalizador de la Universidad de Panamá es reiterado por la Ley 11 de 1981 y esta función está reglamentada mediante resolución del Consejo Académico de la Universidad de Panamá. Por lo que la autorización y regulación de las universidades privadas en Panamá está a cargo de dos instancias en dos actos sucesivos: el acto de aprobación de su fundación como institución por parte del Ministerio de Educación y Presidencia de la República, y el acto de evaluación y aprobación de sus estatutos, planes y programas de estudio, instalaciones físicas y cuerpo docente por parte de la Universidad de Panamá. Fiscalización que se realiza en dos momentos: previa a la aprobación de la solicitud, y continua durante el funcionamiento de las dependencias y las carreras. En 1995 las universidades panameñas (públicas y privadas), a iniciativa del Rector de la Universidad de Panamá, establecieron el Consejo de Rectores de Panamá para: "Procurar en forma permanente, el mejoramiento de la calidad y pertinencia, equidad y modernización de la gestión de las instituciones de educación superior, para lograr una mejor eficiencia y eficacia administrativa". Como parte de su Plan Estratégico, el Consejo se planteó el objetivo de procurar, en forma permanente, el mejoramiento de la calidad, pertinencia y equidad de la educación superior. En este contexto, el Consejo se propuso la creación de un Consejo Nacional de Acreditación de la Educación Universitaria. Recientemente, se logró consensuar un proyecto de Ley creadora del Sistema Nacional de Evaluación y Acreditación para el Mejoramiento de la Calidad de la Educación Superior Universitaria, cuyo máximo organismo es el Consejo Nacional de Evaluación y Acreditación Universitaria de Panamá (CONEAUPA). El proyecto fue aprobado recientemente por la Asamblea Nacional de Panamá.

\subsubsection{Nicaragua:}

Recientemente se constituyó una Comisión de Trabajo con representantes del MEDC, el CNU y el COSEP para elaborar una propuesta de Modelo de Acreditación para Nicaragua. (Segunda fase del préstamo del BID). Con fondos de la primera fase del préstamo del BID, 33 Universidades 
presentaron sus Informes de Autoevaluación y cerca de 20 recibieron las visitas de pares externos y elaboraron sus planes de mejoramiento.

La Asamblea Nacional de Nicaragua aprobó la Ley General de Educación en el mes de agosto de 2006, que contiene un título dedicado a la evaluación y acreditación. Efectivamente, el título VI de dicha ley crea el Consejo Nacional de Evaluación y Acreditación del Sistema Educativo Nacional (CNAE) "como el único órgano competente del Estado de acreditar a las Instituciones Educativas de Educación Superior tanto públicas como privadas, así como evaluar el resultado de los procesos educativos desarrollados por el MEDC y el INATEC". "El Consejo Nacional de Evaluación y Acreditación conformará comisiones nacionales de evaluación y acreditación para cada subsistema educativo, los parámetros, criterios y estándares de calidad aplicables en cada subsistema tanto para evaluación como acreditación deberán ser construidos en consulta con las instituciones legalmente constituidas en cada uno de ellos o su representante".

Esta ley contiene algunas disposiciones que han generado críticas en los sectores académicos, como son las siguientes:

(a) "Es legítimo, para todos los efectos de evaluación y acreditación, que dos o más instituciones sumen sus capacidades y posibilidades físicas materiales o académicas para garantizar el cumplimiento de estándares de calidad instituidas por el sistema, en beneficios de sus alumnos".

(b) "El Consejo Nacional de Evaluación y Acreditación estará integrado por cinco (5) miembros, quienes serán electos por la Asamblea Nacional, a propuesta presentada por las Universidades Públicas, Universidades Privadas, Presidente de la República, Diputados y Asociaciones de Profesionales legalmente constituidas. La Asamblea Nacional elegirá al Presidente y Vice-Presidente de este Organismo, en el mismo acto de selección de los miembros del Consejo".

El Consejo deberá rendir informes a la Asamblea Nacional anualmente.

Con estas disposiciones se estima que se corre el riesgo que el organismo acreditador sea manejado con criterios políticos, desde luego que la Asamblea Nacional, foro eminentemente político, tendrá una gran incidencia en su integración y funcionamiento. El CNAE aún no ha elaborado su reglamento interno y no ha comenzado sus labores de acreditación. 
2.4.7 En la actualidad, existen tres agencias o sistemas regionales de acreditación, cuyo objetivo se centra en la acreditación de programas profesionales específicos y postgrados. Ellos son:

I. La Agencia Centroamericana de Acreditación de Arquitectura y de Ingeniería (ACAAI), que tiene ya aprobado su Convenio Constitutivo. Su sede es Panamá. Cuenta con un Consejo y un Director Ejecutivo y oficinas en la Universidad Tecnológica de Panamá.

II. La Agencia Centroamericana de Acreditación de Sector Agroalimentario y Recursos Naturales (ACESAR), creada en junio de 2005, que ya instaló su Dirección Ejecutiva en Guatemala, y cuya sede será rotativa entre los países centroamericanos.

III. La Agencia Centroamericana para la Acreditación de los Postgrados (ACAP), que cuenta con una Dirección Ejecutiva y tiene su sede en Honduras. Fue creada en agosto de 2006.

Es importante señalar que en estos proyectos están involucrados los respectivos Colegios Profesionales de las carreras antes mencionadas y las Facultades universitarias que las imparten, tanto de las universidades estatales como privadas de la región centroamericana.

En el ámbito de las universidades privadas existen en Centroamérica el "Sistema de Acreditación de Carreras de Enseñanza Superior Universitaria Privada de Costa Rica" (SUPRICORI) y la Agencia de Universidades Privadas de Centroamérica (AUPRICA), creada en 1990 por 16 universidades privadas de El Salvador, Honduras, Nicaragua y Costa Rica. Realiza procesos de evaluación y acreditación institucional.

\subsection{El Consejo Centroamericano de Acreditación de la Educación Superior (CCA).}

El Consejo Centroamericano de Acreditación de la Educación Superior (CCA), fue creado como resultado de los Foros Centroamericanos de Acreditación de la Educación Superior, convocados por el Consejo Superior Universitario Centroamericano (CSUCA), con la colaboración económica de la agencia alemana InWent. Estos Foros reunieron a representantes de las Universidades públicas, delegados de las Universidades privadas con sólido prestigio en la región centroamericana (Belice, Guatemala, Honduras, El Salvador, Nicaragua, 
Costa Rica y Panamá), a los Ministros de Educación, a representantes de los Colegios Profesionales del área, a delegados estudiantiles y a representantes de las agencias nacionales y regionales de acreditación existentes o en proceso de formación. El acuerdo de creación fue adoptado en el II Foro (San José, mayo de 2002) y en el III Foro (Guatemala, octubre de 2002) se aprobó el texto del Convenio de Constitución del CCA, sus Estatutos y presupuesto de instalación y funcionamiento. Una vez electos los miembros del Consejo, estos tomaron posesión en el IV Foro celebrado en Panamá, en noviembre de 2004. Inmediatamente el CCA celebró su primera sesión de trabajo y aprobó su plan estratégico de trabajo.

Entre las razones que se esgrimieron para crear el CCA, cabe mencionar las siguientes:

“- Que la educación superior es imprescindible para el desarrollo de los países centroamericanos y de la región en general.

- Que la visión compartida de los países centroamericanos, constituye el sustento para el logro armónico y coherente de un proyecto de mejoramiento continuo de la calidad de la educación superior centroamericana.

- Que la evaluación y la acreditación de la educación superior son instrumentos para promover el mejoramiento de la calidad de las instituciones de educación superior y de sus programas, así como para ofrecer información pertinente a la sociedad, sobre la calidad de los mismos.

- Que la evaluación y la acreditación son instrumentos eficaces para establecer la pertinencia y promover el impacto social de los programas educativos

- Que el establecimiento de sistemas de aseguramiento de la calidad de la educación superior, es un imperativo como respuesta al contexto de la globalización económica y social; y que dichos sistemas contribuyen al reconocimiento mutuo de las competencias académicas entre los distintos países y regiones.

- Que es necesario crear un organismo regional, con perspectiva y alcance centroamericano, que defina, regule y aplique principios y normas de buena práctica de la evaluación y la acreditación. 
- Que el reconocimiento a escala regional de la acreditación de la calidad de la educación superior, coadyuvará al reconocimiento internacional de la calidad de títulos y grados, a la movilidad académica y a fortalecer el reconocimiento internacional de la calidad de los programas e instituciones de educación superior acreditados.

- Que en la región se requiere promover y desarrollar sistemas de aseguramiento de la calidad de la educación superior, que cumplan con los principios de buena práctica aceptados internacionalmente y que reconozcan las características propias de cada país y de la región centroamericana".

La iniciativa del CSUCA coincidió, en su finalidad, con la tomada por los Ministros de Educación de Centroamérica, según acuerdo No. 18 de la XXI Reunión Ordinaria de la Coordinación Educativa y Cultural Centroamericana (CECC), realizada en abril de 2002 en Guatemala. Los Ministros de Educación también abogaban por la creación de un organismos regional de acreditación. El CSUCA les invitó a unir esfuerzos, lo cual facilitó la creación del CCA.

El Convenio Constitutivo del CCA creó, al mismo tiempo, una Junta de Coordinación y Seguimiento, integrada por dos representantes de cada uno de los sectores participantes en el Convenio, con las funciones siguientes: a) proveer el apoyo que el CCA requiera para su funcionamiento; b) servir de enlace entre el CCA con los cuatro sectores signatarios del Convenio; c) dar seguimiento al desarrollo y cumplimiento del Convenio; d) atender lo relativo a la reforma del Estatuto; e) revisar los montos de la contribución financiera de los diferentes sectores; f) atender lo relativo a la incorporación de nuevas instituciones suscribientes del Convenio.

El CCA, según el Convenio Constitutivo, es un organismo regional centroamericano sin fines de lucro, constituido por los sectores académicos, público y privado, gubernamental y profesional de Centroamérica, como la instancia encargada de conceder la acreditación y el reconocimiento regional a los organismos de acreditación de programas e instituciones que operen en cada país o en la región. Dicho reconocimiento se otorgará según los lineamientos, características y marcos de referencia para la acreditación que el Consejo defina. La misión del Consejo es promover el mejoramiento de la calidad y la integración de la educación superior centroamericana, a través de agencias nacionales o regionales de acreditación, debidamente reconocidas por el CCA.

El Consejo Centroamericano de Acreditación de la Educación Superior lo forman once profesionales y un estudiante destacado. Siete de los profesionales son designados, uno por cada uno de los países centroamericanos. Cuatro de los 
profesionales son designados a nivel regional, uno por cada uno de los siguientes sectores: académico-estatal, académico-privado, gubernamental y profesional. También es miembro regional un estudiante, designado por el propio Consejo a propuesta de las asociaciones estudiantiles reconocidas en cada país.

El Consejo Centroamericano de Acreditación de la Educación Superior tiene un(a) Director(a) Ejecutivo(a), quien participa en las reuniones y deliberaciones con derecho a voz pero sin voto.

Los miembros del Consejo Centroamericano de Acreditación de la Educación Superior son designados por períodos de cuatro o seis años, a excepción del estudiante quien ejerce el cargo durante dos años. Se renuevan en forma alterna.

La designación de los miembros del Consejo Centroamericano de Acreditación de la Educación Superior por los sectores académico estatal, académico privado, gubernamental, profesional y estudiantil, corresponde al Consejo Superior Universitario Centroamericano, al conjunto de las universidades privadas que son signatarias del Convenio de Creación, a la Coordinación Educativa y Cultural Centroamericana (CECC) al Consejo de Entidades Profesionales Universitarias de Centroamérica (CEPUCA) y a las Asociaciones estudiantiles de las instituciones de educación superior acreditadas.

Al Consejo Centroamericano de Acreditación de la Educación Superior le corresponden, entre otras, las atribuciones siguientes:

“1. Determinar y velar por el estricto cumplimiento de las políticas, procedimientos, criterios y estándares para la evaluación y el reconocimiento regional de agencias acreditadoras formalmente establecidas que funcionan en la región, a tono con los estándares y principios internacionalmente reconocidos, respetando el marco legal de cada país y en consulta con dichas agencias, y velar por su estricto cumplimiento.

2. Otorgar reconocimiento regional a los organismos acreditadores de Educación Superior que funcionen en la región.

3. Respetar y garantizar que los organismos acreditadores, en el desarrollo de sus procesos de acreditación, respeten y preserven la naturaleza y régimen académico y jurídico de las instituciones de educación superior y las leyes, tratados y convenios de cada país.

4. Promover y apoyar la creación de agencias u organismos de acreditación en los países de la región donde no existan; así como organismos regionales especializados de acreditación. 
5. Coadyuvar al fortalecimiento y transformación de los organismos de acreditación que no cumplan con los requisitos para su reconocimiento.

6. Promover el intercambio y colaboración entre los organismos de acreditación que reconozca.

7. Promover alianzas estratégicas y acuerdos de colaboración, intercambio y reconocimiento mutuo con agencias de acreditación de fuera de la región y redes internacionales de acreditación y aseguramiento de la calidad. Sin detrimento de los que las agencias de acreditación de la región puedan establecer por si mismas".

Según sus Estatutos, el Consejo Centroamericano de Acreditación de la Educación Superior elige entre sus miembros un Presidente, dos Vicepresidentes y un Vocal, quienes ejercen el cargo durante dos años, pudiendo ser reelectos. El Secretario del CCA es el Director Ejecutivo.

La Secretaría Técnica es el órgano ejecutivo del Consejo Centroamericano de Acreditación de la Educación Superior. Está integrada por el(la) Director(a) Ejecutivo(a), quien la dirige, y el personal profesional y técnico que se requiera.

El Consejo Centroamericano de Acreditación de la Educación Superior sesiona ordinariamente dos veces al año y extraordinariamente cuando lo convoque el Presidente, a solicitud de más de la mitad de los miembros. Una vez al año y coincidente con alguna de las sesiones ordinarias, el Consejo Centroamericano de Acreditación de la Educación Superior habrá de sesionar conjuntamente con las agencias de acreditación que hayan obtenido su reconocimiento.

Una vez cada dos años y coincidente con una sesión ordinaria, el Consejo Centroamericano de Acreditación de la Educación Superior habrá de sesionar con la Junta de Coordinación y Seguimiento del Convenio de creación y funcionamiento del CCA.

Una vez cada tres años y coincidente con una sesión ordinaria, el Consejo Centroamericano de Acreditación de la Educación Superior habrá de participar en la reunión del Foro Centroamericano por la Acreditación de la Educación Superior.

El Foro Centroamericano por la Acreditación de la Educación Superior es el órgano consultivo del Consejo. Además, es un espacio regional de intercambio de experiencias, reflexión y debate sobre la calidad y pertinencia de la educación superior. Se integra con la representación de: el Consejo Superior Universi- 
tario Centroamericano, universidades privadas suscriptoras del convenio, la Coordinación Educativa y Cultural Centroamericana, el Consejo de Entidades Profesionales Universitarias de Centroamérica, tres estudiantes, las agencias de acreditación de educación superior que funcionen en la región, organismos reguladores de la Educación Superior de los países centroamericanos, el sector empleador de los graduados universitarios y de expertos invitados.

El Foro Centroamericano por la Acreditación de la Educación Superior se reune ordinariamente cada tres años, y es convocado por el CCA, para conocer su opinión sobre los asuntos sometidos a su consideración.

El CCA, en su primera sesión de trabajo, definió su Misión y Visión en los términos siguientes:

"Misión: Contribuir al mejoramiento continuo de la calidad, pertinencia y armonización de la educación superior en Centroamérica, mediante la acreditación y el reconocimiento formal de organismos o agencias de acreditación de instituciones, programas o carreras existentes en cada país o en la región.

Visión: Liderar la integración de un sistema centroamericano de evaluación y acreditación de la educación superior, que articule las capacidades e intereses de los sectores y países que conforman el CCA, mediante la promoción y desarrollo de procesos de mejoramiento continuo de la calidad en los organismos o agencias de acreditación que operen en la región, y en consecuencia de la calidad de la educación superior en Centroamérica".

El primer asunto que el CCA ha tenido que atender se refiere a la obtención de su personalidad jurídica en Costa Rica, requisito indispensable para poder suscribir convenios y contratos, así como para abrir una cuenta bancaria en dicho país y estar en capacidad de disponer de los recursos económicos ya puestos a su disposición. Para tal efecto, se creó la Fundación pro Consejo Centroamericano de Acreditación de la Educación Superior, cuya personalidad jurídica ya fue aprobada. Ahora, como segunda etapa se está gestionando la personalidad jurídica del CCA como organismo regional centroamericano especializado. Los Ministros de Educación de Centroamérica ya firmaron un acuerdo reconociendo esa personalidad y el carácter de organismo oficial centroamericano responsable de la acreditación de agencias nacionales, regionales y extranjeras que ofrezcan servicios de acreditación en la subregión centroamericana. Se está tramitando también, ante la Secretaría General del 
Sistema Centroamericano de Acreditación (SICA), la inscripción del CCA como Organismo No Gubernamental de Consulta del CCA.

En el ámbito técnico, el CCA tiene ya aprobados oficialmente y consultados con las Agencias Nacionales ya existentes y el Foro Centroamericano por la Acreditación de la Educación Superior Centroamericana, los instrumentos técnicos siguientes:

- El Manual de Acreditación: contiene el marco conceptual y el modelo de acreditación del CCA.

- La Guía de Evaluación: contiene los componentes por evaluar, los criterios de calidad, requisitos para la acreditación y pautas de cumplimiento.

- El Reglamento de Acreditación: propone la normativa que orienta el proceso y los lineamientos para los distintos actores involucrados.

- El Código de Ética: presenta los compromisos y responsabilidades de los miembros del Consejo y algunas disposiciones para la solución de conflictos de interés.

- El Glosario: aporta 183 términos relacionados con el proceso de acreditación.

- Instrumentos para la Evaluación Externa: contiene la guía metodológica para la autoevaluación de agencias y otras cinco guías para la evaluación externa y elaboración de informes.

El CCA ya ha empezado a establecer contactos con organismos homólogos de América Latina, como por ejemplo, el COPAES de México. El CCA ya se incorporó a la Red Iberoamericana para la Acreditación (RIACES) y forma parte de su Junta Directiva. También ya fue aceptado por la Internacional Network for Quality Assurance Agencies in High Education (INQAAHE).

El CCA ya está recibiendo cooperación técnica del COPAES, de una Agencia de Cooperación Alemana, el Servicio Alemán de Intercambio Académico (DAAD) y la Conferencia de Rectores de Alemania (HRK), que han cofinanciado algunas de sus actividades. Mantiene una coordinación estrecha de trabajo con el Consejo Superior de Universidades Centroamericanas (CSUCA) y la Coordinadora Educativa y Cultural de Centroamérica (CEEC), que la forman los Ministros de Educación y Cultura de Centroamérica. 


\section{Conclusiones.}

No cabe duda que la acreditación de la calidad de la educación superior, realizada y respaldada a escala centroamericana, resulta más confiable y respetable para la comunidad internacional dedicada a la acreditación y aseguramiento de la calidad de la educación superior y coincide con la visión de una Centroamérica con identidad de región, que es cada vez más difundida hoy día en el mundo en lugar de la vieja visión de un aglomerado geográfico de pequeños países, con los que hay que tratar siempre por separado.

La acreditación realizada a escala regional implica un proceso de construcción de consensos sobre criterios, indicadores y estándares de calidad comunes en la región, lo cual contribuye a la búsqueda de una visión compartida y de horizontes comunes para el mejoramiento y desarrollo de la educación superior de América Central.

Para avanzar en la integración de la educación superior centroamericana, es necesario que existan medios para convalidar en los siete países las acreditaciones de calidad de la educación superior.

Promover el mejoramiento de la calidad y la integración de la educación superior centroamericana es promover la aceleración del desarrollo integral de la región. Es también una estrategia apropiada para diseñar respuestas conjuntas, comunes y coordinadas a los desafíos que plantea el actual proceso de globalización en el campo de la educación superior.

El reconocimiento a escala regional de la acreditación de la calidad de la educación superior, coadyuvará al reconocimiento internacional de la calidad de títulos y grados, a la movilidad académica y a fortalecer el reconocimiento internacional de la calidad de los programas e instituciones de educación superior acreditados.

\section{Bibliografía}

Alarcón Alba, Francisco y Luna, Julio Guillermo: Antecedentes, Situación Actual y perspectivas de la evaluación y acreditación de la educación superior en Centroamérica, Secretaría Permanente del CSUCA, Guatemala, mayo de 2003 / (fotocopiado).

Consejo Nacional de Acreditación - Ministerio de Educación Nacional: La evaluación externa en el contexto de la acreditación en Colombia, Santafé de Bogotá, 1998. 
Knight, Jane: "El comercio de la educación superior. Nuevas oportunidades y riesgos potenciales" en revista Foreign Affairs en español, Vol. 5 Núm. 2, 2005.

Martínez, Eduardo y Letelier, Mario (Editores): Evaluación y Acreditación Universitaria - Metodologías y Experiencias, UNESCO - OUI, Universidad de Santiago de Chile, Editorial Nueva Sociedad, Caracas, 1997.

Orozco Silva, Luis Enrique: Elementos para la creación de un Sistema Nacional de Acreditación, Universidad de Los Andes, Magíster en Dirección Universitaria, M.D.U., Santafé de Bogotá, 1996.

Tünnermann Bernheim, Carlos: Universidad y Sociedad-Balance histórico y perspectivas desde Latinoamérica, Universidad Central de Venezuela, 2000.

UNESCO: Documento de Política para el Cambio y el Desarrollo en la Educación Superior, París, 1995.

UNESCO-CRESALC: Acreditación Universitaria en América Latina - Antecedentes y Experiencias, UNESCO-CRESALC-CINDA, Caracas, 1994.

Yarzábal, Luis, Vila, Ana y Ruiz, Roberto (Editores): Evaluar para transformar, Colección Respuestas No 10, Editores IESALC-UNESCO, Caracas, 1999. 